\title{
Twitter y la organización partidista local durante la elección estatal de Coahuila, 2017
}

Twitter and local party organization during the Coahuila state election, 2017

Antonio Corona y Brenda Azucena Muñoz

Universidad Autónoma de Coahuila (México) antoniocorona@uadec.edu.mx; brenda.munoz@uadec.edu.mx

\section{Resumen}

El presente trabajo consiste en un análisis del tipo y origen de las publicaciones en Twitter en el período de mayor actividad de la elección estatal de Coahuila, México (2017) mediante una recolección sistemática de menciones a candidatos presentes en la boleta. Retomando las cuentas con menciones a partidos políticos en su descripción de usuario, se categorizaron de acuerdo a orígenes gubernamentales o partidistas de los ámbitos local y nacional. Se encontró que las instancias locales del PRI, partido en el poder, representan la mayoría del volumen de publicación a favor del partido, a diferencia de los partidos de oposición, que cuentan con un número mayor de publicaciones por parte de ciudadanos no afiliados. Estos resultados se articulan con las investigaciones existentes sobre los mecanismos tradicionales de movilización 
a nivel local utilizados históricamente por el partido oficialista. A partir de esta articulación se concluye que la aparente popularidad del PRI en Twitter no es, como se había planteado hasta el momento, resultado de un manejo innovador ni de mecanismos de publicación automática (bots), sino que es una extensión de la organización y la distribución de recursos a nivel local que dicho partido ha empleado históricamente para menoscabar las voces disidentes.

Palabras clave: Twitter, elecciones; identidad política; México.

\section{Abstract}

The present work is an analysis of the type and origin of Twitter posts during the most active period (2017) state election in Coahuila, Mexico, through a systematic collection of mentions to candidates in the ballot. The accounts with explicit mentions to political parties in their user description were categorized according to party or government origin both locally and nationally. The local instances of the incumbent PRI were found to represent most of the posting volume in favor of the party, in contrast to higher volumes of unaffiliated citizen support from the opposition parties. These results are articulated with existing research about the mechanisms for local mobilization historically employed by the official party. From this articulation, we conclude that the PRI's seeming popularity isn't the result of better social network management or bot posting, but rather an extension of the mobilization mechanisms that the party has historically used to undermine dissident voices.

Keywords: Twitter; elections; political identity; México.

El 4 de junio de 2017 el estado de Coahuila, una de las treinta y dos entidades federales de México, realizó su vigésimo novena elección para la gubernatura. A nivel nacional, estos comicios fueron momentáneamente eclipsados por la elección para la gubernatura del Estado de México, realizada durante la misma jornada, debido al peso político y económico de dicha entidad, y al hecho de que la elección para gobernador del Estado de México fue tratada en los medios como una predicción de la elección presidencial del año siguiente (Núñez, 2017; Semple, 2017). No obstante, las elecciones de Coahuila pronto acapararon la atención mediática local y nacional por tratarse de una elección plagada de incertidumbre y denuncias de irregularidades (Guardiola, 2017; Romero, 2017). El Partido Revolucionario Institucional (PRI), titular del poder ejecutivo nacional por setenta y ocho de los últimos noventa años y titular, también, de la gubernatura de Coahuila durante toda la historia moderna del estado, fue 
declarado ganador por el Instituto Electoral Estatal, lo que provocó que los candidatos de oposición tanto de izquierda como de derecha formaran un frente común. Dicho frente llamó a la población a defender el triunfo de Guillermo Anaya (1), el candidato del Partido Acción Nacional (PAN) y principal opositor de Miguel Riquelme (2), candidato postulado por el PRI. EI llamado a la unidad y a la protesta, por parte del Frente por un Coahuila Digno, se hizo principalmente a través de la difusión en medios sociales de catorce agravios y 200 pruebas que significarían la anulación de la elección estatal (Delgado, 2017).

Con cada elección en el país, los medios sociales parecieran ganar más terreno tanto en los planes de campaña de los partidos políticos como en el espectro de posibilidades de agencia de la población. El papel de Twitter en los movimientos de protesta, particularmente \#YoSoy132, ha sido estudiado desde diversas perspectivas (González, 2012; Gun Cuninghame, 2016; Welp, 2014), y pareciera indicar que esta plataforma mediática desempeña un rol cada vez más importante como avenida de discusión política del país. Claro está, el formato de Twitter dista de ser idóneo para el intercambio profundo: decir que el discurso político en Twitter está altamente polarizado quizá sea demasiado conservador. La investigación realizada al respecto hasta la fecha, en diversos escenarios políticos alrededor del mundo, revela un panorama de profundas divisiones partidistas y una marcada escasez de diálogo entre posicionamientos políticos opuestos (Gruzd y Roy, 2014; Conover, Ratkiewicz, Francisco, Goncalves, Flammini y Menczer, 2011). Si bien es posible que esta polarización en el discurso de los ciudadanos politizado en Twitter sea simplemente un reflejo de los argumentos y talking points expresados por los actores políticos de su preferencia, sería ingenuo y reduccionista asumir que los usuarios de Twitter con una alta politización tan solo repiten los argumentos de su actor político preferido, independientemente de sus respectivas circunstancias particulares. La efectividad de las comunicaciones políticas en Twitter ha sido objeto de una cantidad considerable de estudios con resultados diversos, tanto en México (Heras y Díaz, 2016; Salgado, 2013) como en otros países (Aharony, 2011; Grant, Moon y Busby, 2010; Lyons y Veenstra, 2016). Por otro lado, se han realizado también estudios respecto a la identidad en Twitter (Marwick y Boyd, 2010; Walton y Rice, 2013) y, específicamente, sobre los aspectos políticos de la identidad dentro del ambiente de esta plataforma (Alvídrez, 2017; Herdagdelen, Zuo, Gard-Murray y Bar-Yam, 2013).

En lo que respecta al caso de México, ya se mencionó que el uso de plataformas sociales por parte de actores políticos ha sido objeto de varios estudios, que han analizado la actividad de cuentas de candidatos y funcionarios, el uso de redes en momentos electorales y su efectividad (Alvídrez, 2007; Salgado, 2013). Específicamente, se ha encontrado una alta presencia del PRI en Twitter, la cual algunos estudios han atribuido a un buen manejo de redes por parte del partido (Heras y Díaz, 2017), mientras que otros trabajos coinciden con la prensa secular, al atribuirla a grandes cantidades de bots (Salgado, 2013; Verkamp y Gupta, 2013). Dichas 
investigaciones, sin embargo, se han ocupado de la actividad en redes sociales como un fenómeno hasta cierto punto aislado y auto-contenido, sin tener en cuenta la injerencia sobre esta esfera de la organización y las tácticas tradicionales de movilización y homogeneización de opiniones dentro de los partidos.

El presente estudio busca realizar una primera mirada a dicha injerencia, analizando el origen de las publicaciones a favor de los partidos, a la luz del conocimiento existente respecto a la manera en que dichos partidos han organizado históricamente sus campañas electorales. En un país en el cual el partido oficialista frecuentemente hace uso (y abuso) de recursos económicos y sociales para la movilización sistemática de ciudadanos con fines de proselitismo (Cornelius, 2004; Magaloni, 2006), resultaría ingenuo y reduccionista asumir que dichos mecanismos, utilizados por décadas en espacios físicos, no tienen ninguna relación con el manejo del partido en espacios virtuales.

Los contextos locales y regionales resultan de gran importancia para el fin planteado: el análisis de la actividad en Twitter en torno a una elección estatal de importancia menor no sólo nos permite trabajar con cantidades manejables de datos, sino que también posibilita acercarnos a una esfera de discusión más alejada de la lente de las dirigencias y organismos centrales a nivel nacional, dándonos así la posibilidad de observar de modo más aislado la actividad de publicación en el ámbito local. Lo anterior es importante, dado que la movilización de la ciudadanía mediante mecanismos de coerción y patronazgo a nivel local y vecinal -lo que Cornelius (2004) Ilamó "movilización autoritaria"- es un eslabón clave en la estructura del Partido Revolucionario Institucional (Corona, 2012; Cornelius, 2004; Magaloni, 2006; Tosoni, 2006). El análisis de Magaloni (2006) respecto a la utilización del programa gubernamental PRONASOL, como método de castigo y recompensa por el comportamiento electoral a nivel municipal, nos muestra un ejemplo de esta estrategia política en acción.

En lo que respecta al ámbito virtual, el estudio de Heras y Díaz (2016) revela que el PRI dio mucha importancia a las redes sociales como herramienta de campaña a nivel local en 2015, y tuvo una mejor organización en este sentido. En Twitter, específicamente, la disonancia entre el volumen de publicaciones emitidas a favor de los regímenes priistas y el sentimiento general de la población tanto dentro como fuera de la red social ha dado lugar a la especulación por parte de los críticos de PRI, quienes argumentan que el gobierno federal hace uso de redes de publicación automática, a las que se denomina coloquialmente peñabots en honor al Presidente de la República, Enrique Peña Nieto (Salgado, 2013). En el presente texto se plantea la posibilidad de que la eficiencia del PRI en medios sociales digitales (particularmente en Twitter) no sea producto de un mayor poder de identificación con los ciudadanos ni de redes de bots, sino de políticas de organización a nivel regional que hacen uso de la militancia del partido para aumentar el volumen de publicación. 
Para esclarecer lo anterior se realizó una recopilación de tweets referentes a la elección estatal de Coahuila publicados durante la etapa cúspide del período electoral, buscando identificar tanto el origen de las cuentas simpatizantes de los principales partidos en contienda como la actividad y volúmenes de publicación de las mismas. Tomando en cuenta los resultados de la investigación de Heras y Díaz (2016), que encontró que el PRI tiene una mayor organización en redes sociales, se formuló la hipótesis de que si bien el volumen de publicación tanto de publicaciones originales (tweets) como de republicaciones (retweets) sería superior entre los simpatizantes del PRI, una categorización de las cuentas nos permitiría distinguir un alto volumen de publicación entre miembros de la dirigencia partidista, en contraste con un volumen mucho más bajo de publicación entre los simpatizantes ciudadanos del partido. Se supuso también que, debido al relativamente alto nivel de organización en redes sociales del PRI, las publicaciones a favor de dicho partido mostrarían un mayor porcentaje de retweets que las de los partidos de oposición, indicando la posible existencia de mecanismos formales o informales de homologación del discurso entre la militancia virtual. Cabe mencionar que, al referirnos a la dirigencia de los partidos, no nos referimos necesariamente a las altas esferas de cada organización, sino a los militantes con cargos específicos a niveles estatal, municipal y distrital.

\section{Método}

La presente investigación se dio a la tarea de estudiar el origen de las publicaciones a favor de los diversos candidatos en contienda en la elección de gobernador del estado de Coahuila, México, en 2017. Para esto se hizo un análisis de contenido de las descripciones de usuario de las cuentas en cuestión, con la finalidad de identificar enunciaciones tanto a favor como en contra de los partidos políticos en pugna, así como enunciaciones identitarias que revelen el grado de involucramiento político del usuario.

\begin{tabular}{|l|l|l|l|}
\hline \multicolumn{1}{|c|}{$\begin{array}{c}\text { Nombre del } \\
\text { candidato }\end{array}$} & $\begin{array}{c}\text { Cuenta oficial de } \\
\text { Twitter }\end{array}$ & $\begin{array}{c}\text { Partido político de } \\
\text { afiliación }\end{array}$ & $\begin{array}{c}\text { Partidos políticos } \\
\text { en alianza }\end{array}$ \\
\hline Guillermo Anaya & @memo_anaya & PAN & UDC, PPC, PES \\
\hline Miguel Riquelme & @mrikelme & PRI & $\begin{array}{l}\text { PVM, PaNAI, PJ, } \\
\text { PRC, PCP, SI }\end{array}$ \\
\hline Mary Telma Guajardo & @mary_telma & PRD & - \\
\hline Armando Guadiana & @aguadiana & Morena & - \\
\hline José Ángel Pérez & @JoseAngelPerez & PT & - \\
\hline Javier Guerrero & @guerrerogmx & Independiente & - \\
\hline
\end{tabular}


El corpus del presente estudio fue obtenido mediante un proceso de recopilación y selección por etapas. Como primer paso, se utilizó un código programático (script) realizado en el lenguaje Python para recuperar e indizar todos los tweets dirigidos o mencionando a los candidatos a gobernador de Coahuila. Dicho script realizó los registros con una frecuencia diaria mediante el envío de requisiciones de pesquisa (queries) al API de Twitter, utilizando los nombres de usuario de los candidatos (tabla 1) como término de pesquisa. Con la finalidad de realizar una recopilación exhaustiva, se utilizó como condición de finalización del registro la llegada al tweet más reciente registrado el día anterior. Esta recopilación diaria fue realizada desde el miércoles anterior al día de inicio de la veda electoral (24 de mayo y 1 de junio, respectivamente) hasta el miércoles posterior al domingo de jornada electoral (4 de junio), finalizando así la muestra el 14 de junio. Se recuperaron un total de 217.980 tweets, de los cuales se removieron 643 emitidos por las cuentas de los candidatos, permaneciendo un total neto de 35.823 tweets de 9.859 usuarios y 181.514 retweets. Se realizó un análisis de contenido de las descripciones de usuario (Twitter Bio) para identificar menciones positivas o negativas a partidos políticos involucrados en el proceso electoral, y para identificar la situación política de los usuarios, con la siguiente categorización:

\begin{tabular}{|l|l|}
\hline Ciudadano & $\begin{array}{l}\text { La descripción de usuario no menciona afiliación formal a ningún partido } \\
\text { político, ni el desempeño de algún cargo público en la actualidad o en el } \\
\text { pasado. }\end{array}$ \\
\hline Dirigencia local & $\begin{array}{l}\text { La descripción de usuario menciona el desempeño actual o pasado de } \\
\text { un cargo formal en el organigrama de un partido político a nivel estatal, } \\
\text { municipal o distrital, o la candidatura actual o pasada a un cargo público } \\
\text { a este nivel por parte del partido. }\end{array}$ \\
\hline Dirigencia nacional & $\begin{array}{l}\text { La descripción de usuario menciona el desempeño actual o pasado de } \\
\text { un cargo formal en el organigrama de un partido político a nivel } \\
\text { nacional, o la candidatura actual o pasada a un cargo público a este } \\
\text { nivel por parte del partido. }\end{array}$ \\
\hline Gobierno local & $\begin{array}{l}\text { La descripción de usuario menciona el desempeño actual o pasado de } \\
\text { un cargo público o de elección popular a nivel estatal, municipal o } \\
\text { distrital. }\end{array}$ \\
\hline Gobierno nacional & $\begin{array}{l}\text { La descripción de usuario menciona el desempeño actual o pasado de } \\
\text { un cargo público o de elección popular a nivel nacional. }\end{array}$ \\
\hline
\end{tabular}

Tabla 2: Categorización de tipos de cuenta por origen. Fuente: elaboración propia. 
En una posterior etapa se determinó la frecuencia y volumen de tweets y retweets publicados por las cuentas categorizadas mediante estos criterios, con la finalidad de elucidar una comparativa entre la actividad de los ciudadanos sin afiliación formal declarada y la de las dirigencias locales y nacionales de los partidos alrededor de la elección de Coahuila. En la etapa subsiguiente se recopiló la actividad de las cuentas categorizadas durante el período de análisis, dividiéndola por actividad de cuentas simpatizantes de cada partido y tomando en cuenta la proporción entre tweets y retweets en el volumen total. Los datos empíricos fueron principalmente procesados mediante el programa de análisis estadístico IBM-SPSS y los resultados de cada etapa de análisis se muestran a continuación.

\section{Resultados}

Como se puede apreciar en la tabla 3, el análisis de contenido arrojó un total de 660 menciones positivas y 92 menciones negativas a partidos políticos en las descripciones de los 9.859 usuarios que publicaron tweets durante el período estudiado. Respecto a lo mostrado en esta tabla, conviene esclarecer que la misma descripción de usuario puede contener menciones positivas y negativas de distintos partidos. Por ejemplo, un usuario de Twitter puede mencionar en su biografía ser priísta, lo que calificaría de mención positiva, pero también puede presentarse como anti-panista, lo que fue clasificado como mención negativa. Una posterior etapa de análisis de contenido se ocupó de la categorización de estas cuentas en los rubros explicados en la tabla 2. El resultado de esta segunda etapa de análisis se muestra en la tabla 4.

\begin{tabular}{|l|l|l|}
\hline Partido & Mención positiva & Mención negativa \\
\hline PRI & 341 & 38 \\
\hline PAN & 203 & 24 \\
\hline Morena & 80 & 16 \\
\hline PRD & 4 & 13 \\
\hline PT & 2 & 1 \\
\hline TOTAL & 660 & 92 \\
\hline
\end{tabular}

Tabla 3: Mención de partidos políticos en descripciones de usuario. Fuente: elaboración propia. 


\begin{tabular}{|c|c|c|c|c|c|c|}
\hline \multirow[b]{2}{*}{ Tipo de cuenta } & \multicolumn{2}{|l|}{ PRI } & \multicolumn{2}{|l|}{ PAN } & \multicolumn{2}{|l|}{ Morena } \\
\hline & Positiva & Negativa & Positiva & Negativa & Positiva & Negativa \\
\hline Ciudadano & 42 & 38 & 203 & 24 & 80 & 16 \\
\hline Gobierno federal & 6 & 0 & 29 & 0 & 3 & 0 \\
\hline Gobierno local & 25 & 0 & 30 & 0 & 6 & 1 \\
\hline Dirigencia local & 247 & 0 & 85 & 0 & 22 & 0 \\
\hline Dirigencia nacional & 21 & 0 & 36 & 0 & 6 & 0 \\
\hline TOTAL & 341 & 38 & 383 & 24 & 117 & 17 \\
\hline
\end{tabular}

Tabla 4: Comparativo de tipos de cuenta en menciones de PRI, PAN y Morena. Fuente: elaboración propia.

En la categorización por tipo de usuario mostrada arriba se aprecia que, a diferencia de los casos del PAN y Morena, en los cuales los usuarios son mayormente miembros de la ciudadanía, en el caso del PRI los usuarios son mayormente miembros de la dirigencia local. Se puede apreciar también que la diferencia entre menciones positivas y negativas del PAN y Morena es mucho mayor que la del PRI. Los partidos nacionales PRD y PT no fueron incluidos en la comparación anterior ni en las subsiguientes, debido a que la baja ocurrencia de cuentas mencionando a estos partidos -visible en la tabla 3- no nos permite incluirlos en la discusión.

Cabe mencionar que si bien la pesquisa de datos para conformar la muestra fue realizada sobre menciones de la elección estatal de Coahuila, esto no quiere decir que las categorías de gobierno y dirigencia locales se refieran únicamente al ámbito local de este Estado sino que, por el contrario, se categorizaron instancias locales de cualquier parte del país con los criterios mencionados arriba. En la siguiente etapa de la investigación se analizó la totalidad de publicaciones de las cuentas presentadas en la tabla 4, resultando un total de 5.165 publicaciones provenientes de cuentas simpatizantes del PRI; 2.202 publicaciones de cuentas de simpatizantes del PAN y 676 de cuentas simpatizantes de Morena.

En las figuras 1, 2 y 3 se muestra el análisis de volumen de publicaciones por tipo de usuario, dividido por día. Como se puede apreciar, existe un descenso drástico en el volumen de publicación por parte de simpatizantes de todos los partidos entre el 1 y el 4 de junio. Este decremento corresponde al acato de la llamada "veda electoral", un período previo a la elección establecido por las instancias electorales nacional y estatales durante el cual no se permite la publicación de propaganda electoral de ningún tipo (INE, 2017). La veda electoral no restringe la actividad de la población en general. Una comparación entre las figuras 1, 2 y 3 nos muestra que la mayor parte del volumen de tweets publicados a favor del PRI provienen de cuentas pertenecientes a instancias locales de la dirigencia partidista. En el caso del PAN hay un mayor 
equilibrio entre publicaciones de ciudadanos y de dirigentes del partido y en el caso de Morena las publicaciones son mayormente ciudadanas.

También sirve prestar atención al volumen de publicaciones. Como ya se mencionó, hay diferencias importantes en el volumen de publicación: mientras que las publicaciones de cuentas favorecedoras al PRI superaron las 600 instancias en el día de mayor actividad; las cuentas del PAN alcanzaron en su momento pico apenas la mitad de ese número y las cuentas de Morena no superaron los 100 ítems en su día de mayor actividad.

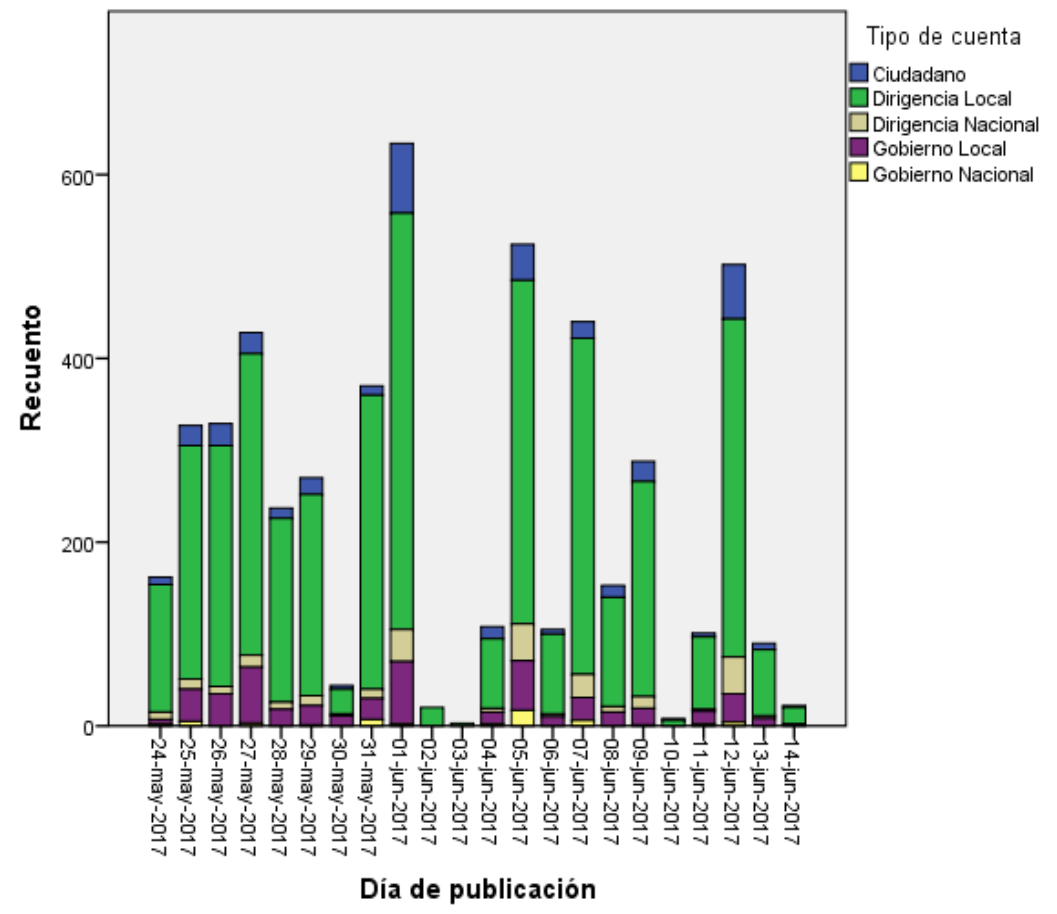

Figura 1: Volumen de publicación por día de cuentas simpatizantes del PRI. Fuente: elaboración propia 


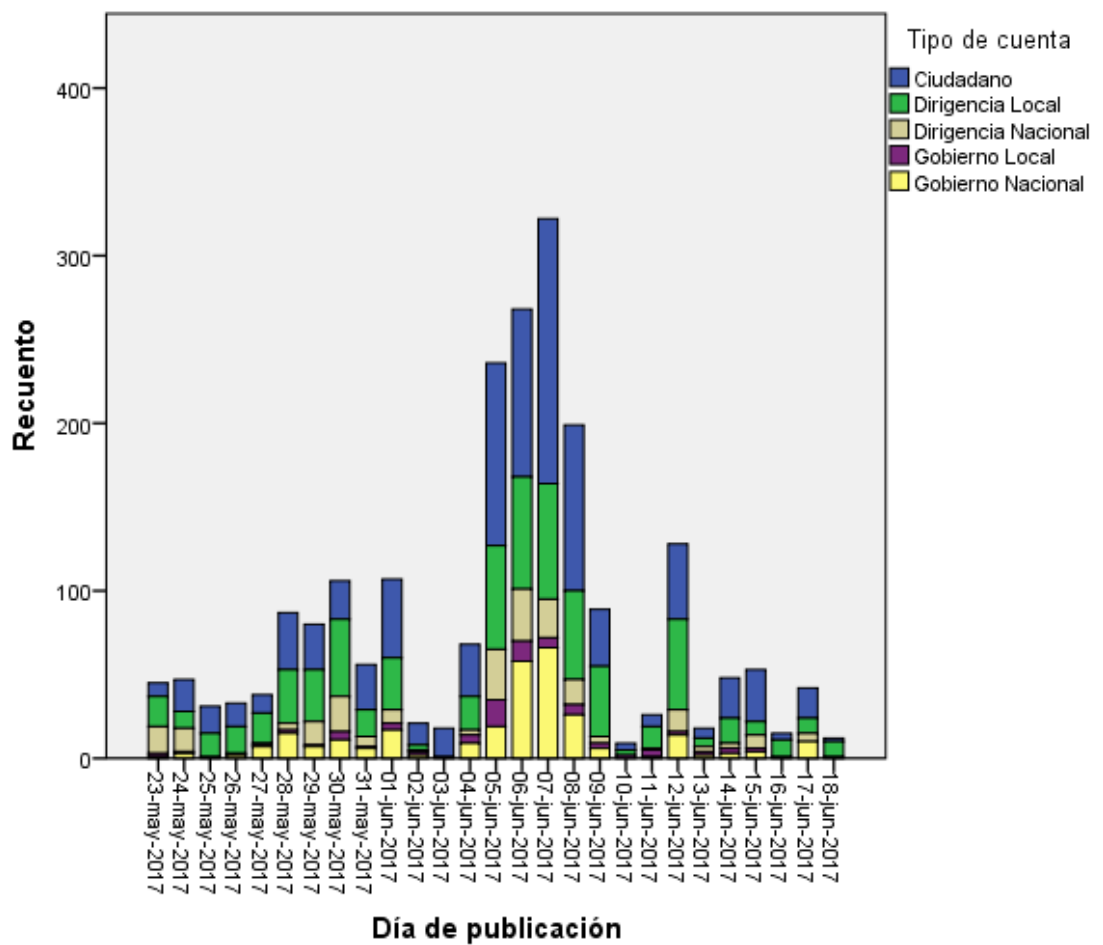

Figura 2: Volumen de publicación por día de cuentas simpatizantes del PAN. Fuente: elaboración propia.

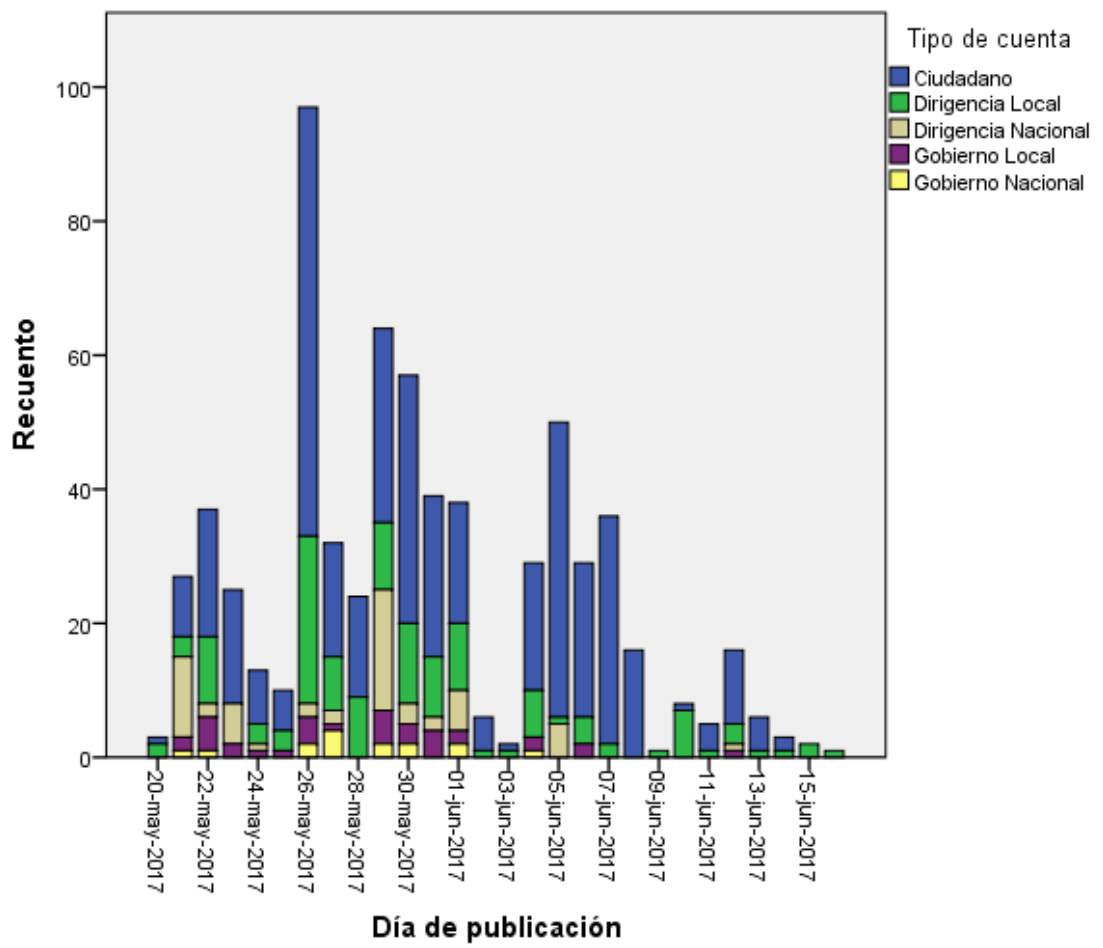

Figura 3: Volumen de publicación por día de cuentas simpatizantes de Morena. Fuente: elaboración propia. 
Hay que recordar que estos números no son de ningún modo indicativos del total de volumen de publicación en Twitter en torno a la elección en Coahuila, sino que solamente corresponden a una intersección muy específica de análisis: las cuentas de Twitter con menciones de partidos políticos en sus descripciones de usuario que mencionaron directamente a alguno de los candidatos a la gubernatura de Coahuila entre el 24 de mayo y el 14 de junio.

En las figuras 4, 5 y 6 se muestra la proporción de tweets originales y retweets en la publicación de las cuentas simpatizantes de los partidos. Como se puede apreciar, la desproporción entre publicaciones originales y republicaciones (retweets) es mayor en el caso de las cuentas simpatizantes del PRI que en el caso de los otros partidos. Si se tiene en cuenta que los tweets originales corresponden a las enunciaciones propias mientras que los retweets son simplemente republicaciones de enunciaciones de otras cuentas, se puede observar en el caso de los tres partidos una uniformidad de enunciaciones que, si bien es un poco más marcada en el PRI, no constituye una diferencia suficiente con los otros partidos ni con el total de las publicaciones que comprendieron la muestra como para constituir un fenómeno aparte.

En la figura 4, sin embargo, se puede apreciar de nuevo la importancia de las instancias locales del partido para la distribución de contenidos.

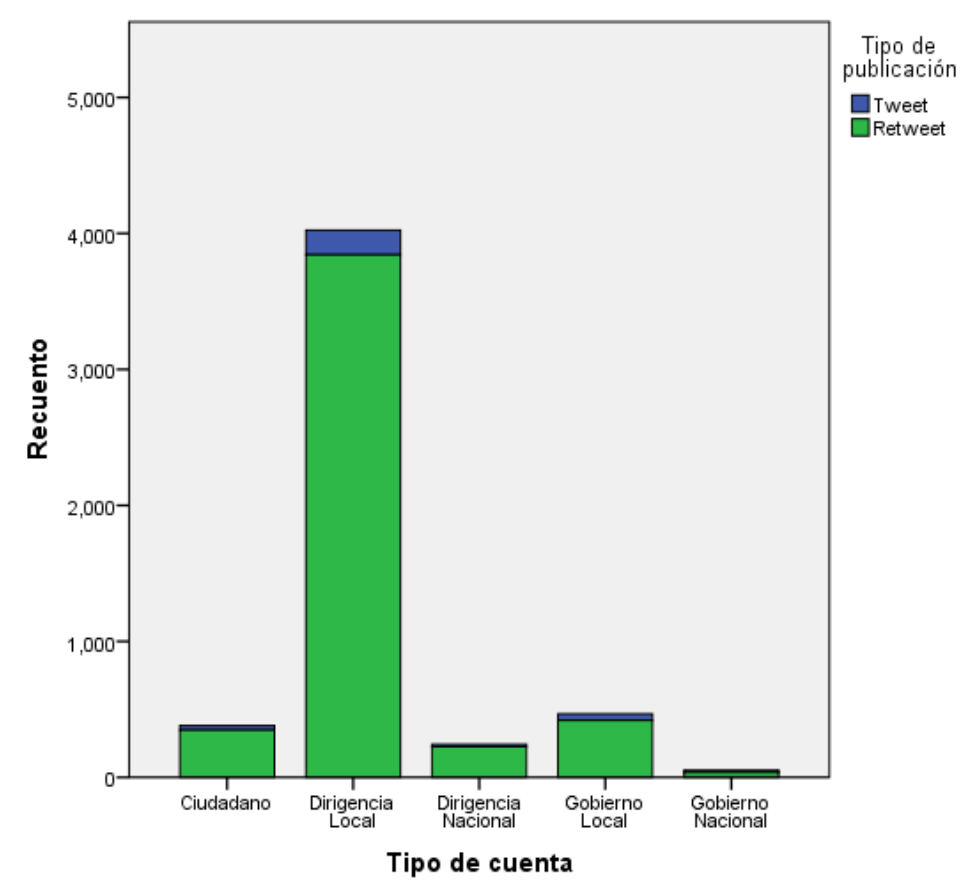

Figura 4: Volumen de publicación de cuentas simpatizantes del PRI. Fuente: elaboración propia. 


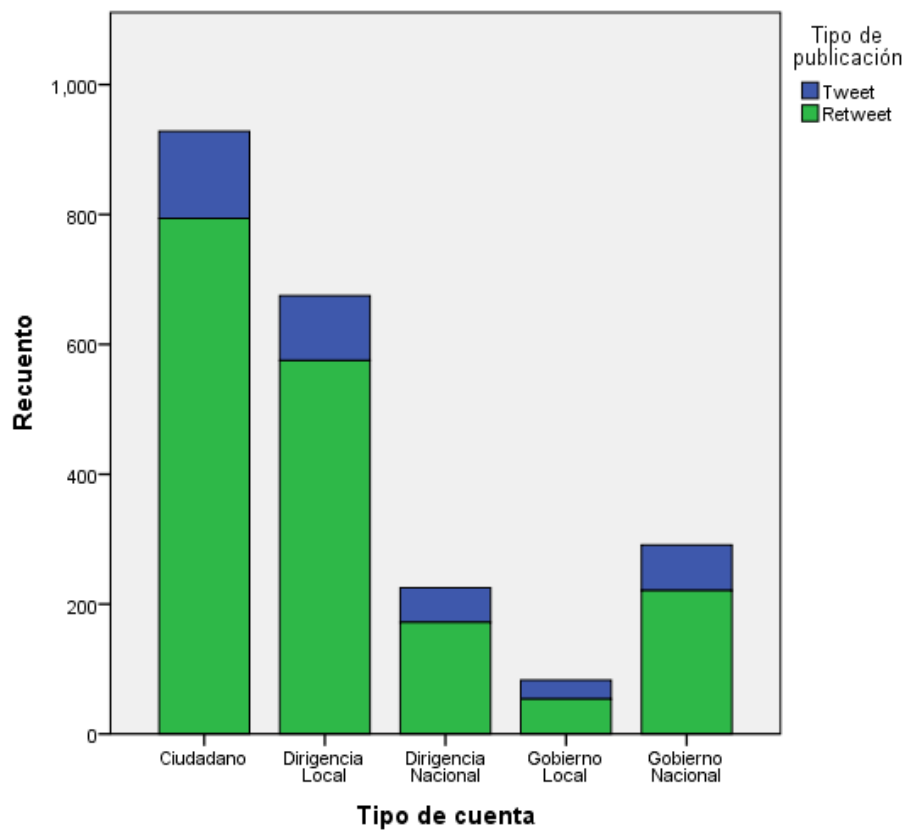

Figura 5: Volumen de publicación de cuentas simpatizantes del PAN. Fuente: elaboración propia.

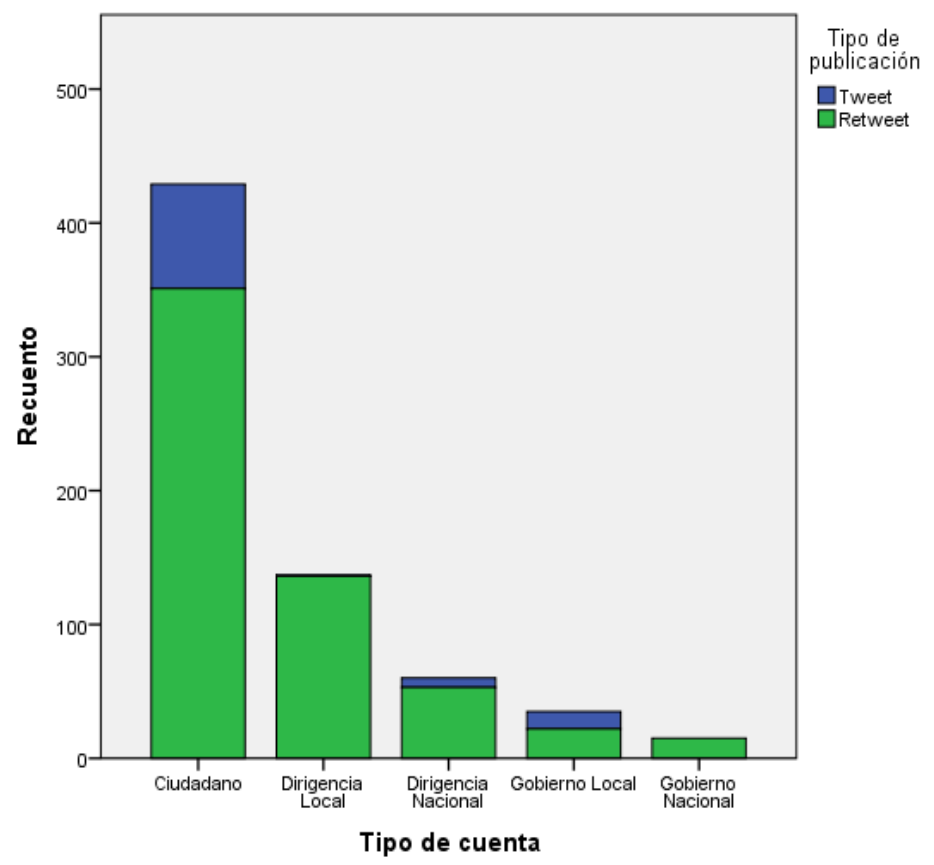

Figura 6: Volumen de publicación de cuentas simpatizantes de Morena. Fuente: elaboración propia.

Como ya se mencionó, la presente investigación partió de dos supuestos: que debido a la mayor capacidad de movilización del PRI en redes sociales a nivel local, derivada de las prácticas históricas de patronazgo por parte del partido en ámbitos municipales, un análisis de cuentas simpatizantes a los partidos durante un período electoral nos mostraría una alta 
actividad de formulación y distribución de mensajes por parte de las organizaciones partidistas locales, en contraste a una relativamente baja actividad de simpatizantes ciudadanos no afiliados al partido. Esta diferencia es fácilmente apreciable en las tablas y figuras que ya se presentaron. El segundo supuesto fue que se encontraría una mayor uniformidad de enunciaciones (un mayor número de retweets y menor de tweets originales) entre las cuentas simpatizantes del PRI. En lo que respecta a este supuesto, si bien la diferencia existe, no es significativa y, por lo demás, tampoco hay una diferencia significativa en la proporción tweet/retweet entre las cuentas simpatizantes de partidos y el resto de las cuentas que formaron parte de la muestra. Los resultados empíricos que mostramos aquí contribuyen al esclarecimiento del papel de la organización local en las campañas electorales en ámbitos virtuales.

\section{Conclusiones}

A partir de los resultados, podemos observar una alta participación política por parte de miembros de la militancia del PRI en Twitter. La avasallante prevalencia de la actividad de dirigentes locales del PRI de todo el país en la discusión de una gubernatura que territorialmente no los involucra indica que la organización política vecinal de dicho partido y sus mecanismos de movilización se extienden a ámbitos virtuales.

En ese sentido, conviene analizar la actividad del PRI en redes sociales no como un fenómeno aislado -como hasta el momento se ha hecho-, sino como una extensión de los mecanismos éticamente discutibles de organización a nivel local que el partido ha empleado durante décadas (Cornelius, 2004; Magaloni, 2006), y que han sido su carta fuerte en contiendas electorales.

Los resultados de este estudio indican que la difusión masiva de contenidos en Twitter por parte del PRI no debe ser analizada como un fenómeno aislado, sino como una consecuencia de la organización -y distribución de recursos federales- en liderazgos vecinales con fines de proselitismo económicamente motivado, o "movilización autoritaria" (Cornelius, 2004), de la cual ya se ha hablado arriba.

Resulta clave para analizar el manejo de redes sociales del PRI, no comprender a este como un fenómeno nuevo nacido de la era digital, sino como una extensión de las políticas problemáticas que han sido utilizadas históricamente por el partido. De no hacerlo, corremos el riesgo de caer en la ignorancia voluntaria al obviar el conocimiento existente, y bautizar como nuevos fenómenos a realidades que son producto de contextos sociohistóricos ampliamente estudiados. 
Dicho lo anterior, habría que hacer dos precisiones: primero, ejercer mucha cautela antes de considerar la muestra aquí analizada como representativa del grueso de la actividad en Twitter porque, como ya se discutió, las cuentas abiertamente simpatizantes de partidos políticos constituyeron tan solo una pequeña fracción del total de la actividad en torno al tema de las candidaturas para la elección. El siguiente paso en el estudio de este fenómeno sería tal vez la realización de sociogramas que busquen una correspondencia (o ausencia de ella) entre las enunciaciones en Twitter de la alta dirigencia del partido y la actividad de las cuentas locales. En este sentido, futuras investigaciones podrían comparar, también, el grado y la forma de penetración que tienen los contenidos difundidos en Twitter en estos dos niveles de organización partidista local e incluso entre los ciudadanos que no se presentan como simpatizantes de partidos políticos.

La segunda precisión consiste en ejercer igual cautela al declarar una correlación directa entre la discusión política dentro de Twitter y el desempeño electoral de los partidos y los candidatos, los resultados de la investigación exponen cómo un partido político, mediante una eficiente organización a nivel local, puede simular en Twitter un nivel de popularidad que, aparentemente, no corresponde con la mínima diferencia de votos reportados en los resultados de las elecciones (Gudiño, 2017) que posteriormente fueron impugnadas (Delgado, 2017). En ese sentido, debemos preguntarnos sobre la aparente efectividad del mensaje del partido en medios sociales. A esta cuestión, se suman los estudios previos realizados respecto al diálogo político en Twitter, que han revelado que dicho diálogo es pobre y sufre de extrema polarización. Por tal motivo, sería conveniente indagar sobre la manera en que estos mecanismos para la difusión masiva de contenidos e información política se podrían traducir en convencimiento o cambio de opiniones.

En cualquier caso, es necesario no quedarnos solamente en el análisis de la efectividad del uso de medios sociales por parte de los diversos organismos políticos, sino adoptar posiciones críticas y contextualizar nuestra actividad académica con una adecuada problematización de los fenómenos que observamos. Porque, finalmente, ese es el propósito que se persigue en una campaña electoral: convencer al electorado de que el candidato propuesto es la mejor opción para desempeñar cargos directivos del gobierno.

\section{Notas}

(1) La candidatura de Anaya fue apoyada por la coalición electoral llamada Alianza Ciudadana por Coahuila, que incluyó a los partidos locales Unidad Democrática de Coahuila (UDC), Partido Primero Coahuila (PPC) y Partido Encuentro Social (PES).

(2) Del mismo modo, seis partidos más se unieron con el PRI para la candidatura de Riquelme en una alianza electoral denominada Coalición por un Coahuila Seguro: los integrantes de esta coalición fueron, además del PRI, los partidos 
locales Socialdemócrata Independiente (SI), Partido Joven (PJ), Partido de la Revolución Coahuilense (PRC) y Partido Campesino Popular (PCP), además de los partidos nacionales Partido Verde de México (PVM) y Partido Nueva Alianza (PaNAl).

\section{Bibliografía}

Aharony, N. (2012). Twitter use by three political leaders: an exploratory analysis. Online Information Review, 36(4), pp. 587-603.

Alvídrez, S. (2017). Interactividad en Twitter: el efecto de la identidad partidista sobre la evaluación de candidatos políticos y sobre sus intenciones de voto. Comunicación y Sociedad, 26(29), pp. 117-137.

Barba, G. (3 de enero de 2017). El presidente quiere hundir al PRI. Forbes. Recuperado de https://www.forbes.com.mx/el-presidente-quiere-hundir-al-pri/

Conover, M.; Ratkiewicz, J.; Francisco, M.; Gonçalves, B.; Menczer, F. y Flammini, A. (2011). Political polarization on twitter. ICWSM, 133, pp. 89-96.

Cornelius, W. (2004). Mobilized Voting in the 2000 Elections: The Changing Efficacy of Vote Buying and Coercion in Mexican Electoral Politics. En Domínguez, J. y Lawson, C. (eds.). Mexico's Pivotal Democratic Election: Campaign Effects and the Presidential Race of 2000. Stanford, California: Stanford University Press.

Corona Armenta, G. (2012). Las elecciones locales en el Estado de México: la plataforma política de Enrique Peña Nieto a nivel nacional (2009-2011). El Cotidiano, 171, pp. 2939.

Delgado, A. (13 de noviembre de 2017). El Frente por un Coahuila Digno pide al TEPJF anulas elecciones para no dar "continuidad a un gobierno corrupto". Proceso. Recuperado de http://www.proceso.com.mx/510970/frente-coahuila-digno-pide-al-tepjf-anularelecciones-dar-continuidad-a-gobierno-corrupto

González, L. (2012). YoSoy132. Participación política 2.0 en México. Diálogo Político, 29(3), pp. 77-104.

Grant, W.; Moon, B. y Busby, J. (2010). Digital dialogue? Australian politicians' use of the social network tool Twitter. Australian Journal of Political Science, 45(4), pp. 579-604.

Gruzd, A. y Roy, J. (2014). Investigating political polarization on Twitter: A Canadian perspective. Policy \& Internet, 6(1), pp. 28-45.

Guardiola, M. (17 de junio de 2017). Presentan más de 100 impugnaciones por elecciones en Coahuila. El Financiero. Recuperado de http://www.elfinanciero.com.mx/nacional/presentan-mas-de-100-impugnaciones-porelecciones-en-coahuila.html 
Gudiño, A. (8 de junio de 2017). Termina cómputo de votos en Coahuila; resultados dan triunfo a Riquelme. Excelsior. Recuperado de http://www.excelsior.com.mx/nacional/2017/06/08/1168650

Gun Cuninghame, P. (2017). \#YoSoy132 and the 'Mexican Spring'of 2012: Between Electoral Engagement and Democratisation. Bulletin of Latin American Research, 36(2), pp. 192205.

Herdagdelen, A., Zuo, W., Gard-Murray, A. y BarYam, Y. (2013). An exploration of social identity: The geography and politics of newssharing communities in twitter. Complexity, 19(2), pp. 10-20.

Instituto Nacional Electoral (2017). Reglamento de Elecciones. Instituto Nacional Electoral, Ciudad de México. Recuperado de https://www.ine.mx/reglamento-de-elecciones/

Lyons, B. y Veenstra, A. (2016). How (not) to talk on Twitter: Effects of politicians' tweets on perceptions of the Twitter environment. Cyberpsychology, Behavior and Social Networking, 19(1), pp. 8-15.

Magaloni, B. (2006). Voting for autocracy: Hegemonic party survival and its demise in Mexico. Cambridge: Cambridge University Press.

Marwick, A. y Boyd, D. (2011). I tweet honestly, I tweet passionately: Twitter users, context collapse, and the imagined audience. New media \& society, 13(1), pp. 114-133.

Núñez, E. (4 de junio de 2017). Ensayo del 2018. Reforma. Recuperado de http://www.reforma.com/aplicacioneslibre/articulo/default.aspx?id=1128212\&md5=15ca 85

Romero, R. (16 de junio de 2017). Consejero del INE revela irregularidades en elecciones de Coahuila. Vanguardia. Recuperado de http://www.vanguardia.com.mx/articulo/consejero-del-ine-revela-irregularidades-enelecciones-de-coahuila

Salgado, E. (2013). Twitter en la campaña electoral de 2012. Desacatos, 42, pp. 217-232.

Semple, K. (6 de mayo de 2017). La disputa electoral para gobernar el Estado de México y sus implicaciones presidenciales. The New York Times. Recuperado de https://www.nytimes.com/es/2017/05/06/la-disputa-electoral-para-gobernar-el-estadode-mexico-y-sus-implicaciones-presidenciales/

Tosoni, M. (2007). Notas sobre el clientelismo político en la ciudad de México. Perfiles latinoamericanos, 15(29), pp. 47-69.

Verkamp, J. y Gupta, M. (2013). Five Incidents, One Theme: Twitter Spam as a Weapon to Drown Voices of Protest. Ponencia presentada en 3rd USENIX Workshop on Free and Open Communications on the Internet (FOCl'13). 
Walton, S. y Rice, R. (2013). Mediated disclosure on Twitter: The roles of gender and identity in boundary impermeability, valence, disclosure, and stage. Computers in Human Behavior, 29(4), pp. 1465-1474.

Welp, Y. (2014). The Mexican Movement \#yosoy132. Ponencia presentada en Social Protest and Democratic Responsiveness: Realities in Latin America and the Caribbean and the European Union, GIGA (volumen 10).

Zuckermann, L. (7 de julio de 2016). Peña y el PRI en picada. Excelsior. Recuperado de http://www.excelsior.com.mx/opinion/leo-zuckermann/2016/07/07/1103445 